\title{
Immunogenicity of one-dose quadrivalent human papillomavirus vaccine in girls
}

\begin{abstract}
There is now a lot of discussion on less than three doses for Human Papillomavirus (HPV) vaccination and, most probably, the immunity for an "aluminum-only" adjuvanted vaccine would be weaker. The HPV "late" L1 antigen seems to be not of good quality for the quadrivalent vaccine, and therefore L1 should not be a basis for argumentation in favor of a one-dose. We really doubt a "one-dose" proposal to be acceptable for HPV lesion control; certainly, it could be possible for lower response antigens like HPV 18, 11, and 6 with an aluminum-based formulation only. More importantly, the message suggested for a one-dose could well be a very bad one as the waning of immunity could have important clinical implications.
\end{abstract}

Keywords: human papillomavirus, vaccine, immunogenicity
Volume 2 Issue 2 - 2016

\author{
Franco Borruto,' Ciro Comparetto 2 \\ 'Department of Obstetrics and Gynecology, Monaco \\ ${ }^{2}$ Division of Obstetrics and Gynecology, City Hospital, Italy
}

\begin{abstract}
Correspondence: Ciro Comparetto, Division of Obstetrics and Gynecology, City Hospital,Azienda USL Toscana Centro,Via Suor Niccolina 20, 59100 Prato, Italy, Tel +393474856799, Fax +390574802955, Email cicomp@tin.it
\end{abstract}

Received: May 15, 2016 | Published: May 26, 2016
Abbreviations: HPV, human papillomavirus; AS04, adjuvant system 04; MMR, measles, mumps, and rubella; CMI, cell-mediated immunity; Th1-2, thelper 1-2

\section{Introduction}

We all know that there is already a lot of discussion on less than three doses for Human Papillomavirus (HPV) vaccination and, most probably, the immunity for an "aluminum-only" adjuvanted vaccine would be weaker. In a recent paper, ${ }^{1}$ a comparison has been made between a one-dose Gardasil (Merck Sharp \& Dohme, Whitehouse Station, NJ, USA) and the two-three doses one. The data with Cervarix (GlaxoSmithKline Biologicals, Rixensart, Belgium), as adjuvant system 04 (AS04) results in a significant adjuvantation phenomenon, should be documented for comparative reasons.

The HPV "late" L1 antigen seems to be not of good quality for the quadrivalent vaccine, and therefore L1 should not be a basis for argumentation in favor of a one-dose as the author's state.

Furthermore, comparing the immune response with an aviral vaccine is inappropriate, as we know that viral vaccines in the last decades needed boosters that were not expected (see Measles, Mumps, and Rubella [MMR] as a three-dose boost - or the Varicella debate needing two doses for effective protection). ${ }^{2,3}$

What is also important is the titer comparison and the avidity of antibodies between the four HPV types. We are more confident for 16 and 18 but much less for the other types, as we know that the immune response is better for 16 and 18 than for 6 and 11. Let's also not forget that the antibody production versus HPV 18 with Gardasil is significantly lower than versus 16 .

Obviously, with the different antibody measurement methods for both HPV vaccines, comparisons are difficult between Gardasil and Cervarix-AS04 adjuvant for the response could be much better than an "old aluminum" vaccine and more versus HPV 18, 6, and 11 too. Also, the cell-mediated immunity (CMI) effect for AS04 could be an important element (if the association is made between AS04 and CMI - if not protection for the Thelper 1-2 [Th1-Th2] profile). ${ }^{4,5}$

Hence, the concept to have (some) CMI effect and to allow for protection (and possibly lesion control if chronic infection is present) is an important immunological effect which should be more documented, if not recalled in this as a general comment. Published or conceptual support is a point we would look for in the Th1-Th2 aspect as compared to aluminum only.

\section{Conclusion}

We really doubt a "one-dose" proposal to be acceptable for HPV lesion control; certainly, it could be possible for lower response antigens like HPV 18, 11, and 6 with an aluminum-based formulation only. ${ }^{6}$ More importantly, the message suggested for a one-dose could well be a very bad one as the waning of immunity could have important clinical implications.

\section{Acknowledgements}

None.

\section{Conflict of interest}

The author declares no conflict of interest.

\section{References}

1. Sankaranarayanan R, Prabhu PR, Pawlita M, et al. Immunogenicity and HPV infection after one, two, and three doses of quadrivalent HPV vaccine in girls in India: a multicentre prospective cohort study. Lancet Oncol. 2016;17(1):67-77.

2. Fiebelkorn AP, Coleman LA, Belongia EA, et al. Measles virus neutralizing antibody response, cell-mediated immunity, and immunoglobulin g antibody avidity before and after receipt of a third dose of measles, mumps, and rubella vaccine in young adults. $J$ Infect Dis. 2016;213(7):1115-1123.

3. Lev-Tov H. A booster for the varicella vaccine. $\mathrm{Br} J$ Dermatol. 2015;173(5):1120-1121

4. Einstein MH, Levin MJ, Chatterjee A, et al. Comparative humoral and cellular immunogenicity and safety of human papillomavirus (HPV)16/18 AS04-adjuvanted vaccine and HPV-6/11/16/18 vaccine in healthy women aged 18-45years: follow-up through Month 48 in a Phase III randomized study. Hum Vaccin Immunother. 2014;10(12):3455-3465. 
5. Leung TF, Liu AP, Lim FS, et al. Comparative immunogenicity and safety of human papillomavirus (HPV)-16/18 AS04-adjuvanted vaccine and HPV-6/11/16/18 vaccine administered according to 2- and 3-dose schedules in girls aged 9-14years: Results to month 12 from a randomized trial. Hum Vaccin Immunother. 2015;11(7):1689-1702.
6. Caskey R, Andes S, Walton SM. HPV vaccine: Less is more. Vaccine. 2016;34(16):1863-1864. 